OPEN ACCESS

Edited by: Jing-Yan Han,

Peking University, China

Reviewed by:

Houzao Chen,

University of Chinese Academy of Sciences, China

Huabo Su,

Augusta University, United States

${ }^{*}$ Correspondence:

Minzhou Zhang

minzhouzhang@aliyun.com

Specialty section: This article was submitted to Vascular Physiology, a section of the journal

Frontiers in Physiology

Received: 30 October 2017 Accepted: 02 May 2018

Published: 22 May 2018

Citation:

Mao S, Chen P. Li T, Guo L and Zhang M (2018) Tongguan Capsule Mitigates Post-myocardial Infarction Remodeling by Promoting Autophagy and Inhibiting Apoptosis: Role of Sirt1. Front. Physiol. 9:589. doi: 10.3389/fphys.2018.00589

\section{Tongguan Capsule Mitigates Post-myocardial Infarction Remodeling by Promoting Autophagy and Inhibiting Apoptosis: Role of Sirt1}

\author{
Shuai Mao ${ }^{1,2,3}$, Peipei Chen ${ }^{1,2}$, Ting Li ${ }^{1,2}$, Liheng Guo ${ }^{1,2}$ and Minzhou Zhang ${ }^{1,2 *}$ \\ ${ }^{1}$ Key Discipline of Integrated Chinese and Western Medicine, Second Clinical College, Guangzhou University of Chinese \\ Medicine, Guangzhou, China, ${ }^{2}$ Department of Critical Care Medicine, Guangdong Provincial Hospital of Chinese Medicine, \\ Guangzhou, China, ${ }^{3}$ Cardiovascular Institute, Stanford University School of Medicine, Stanford, CA, United States
}

Left ventricular (LV) adverse remodeling and the concomitant functional deterioration contributes to the poor prognosis of patients with myocardial infarction (MI). Thus, a more effective treatment strategy is needed. Tongguan capsule (TGC), a patented Chinese medicine, has been shown to be cardioprotective in both humans and animals following ischemic injury, although its precise mechanism remains unclear. To investigate whether TGC can improve cardiac remodeling in the post-infarct heart, adult C57/BL6 mice underwent coronary artery ligation and were administered TGC or vehicle (saline) for 6 weeks. The results demonstrated that the TGC group showed significant improvement in survival ratio and cardiac function and structure as compared to the vehicle group. Histological and western blot analyses revealed decreased cellular inflammation and apoptosis in cardiomyocytes of the TGC group. Furthermore, TGC upregulated the Atg5 expression and LC3II-to-LC3I ratio but downregulated autophagy adaptor p62 expression, suggesting that TGC led to increased autophagic flux. Interestingly, with the administration of 3-methyladenine, an autophagy inhibitor, in conjunction with TGC, the aforesaid effects significantly decreased. Further mechanistic studies revealed that TGC increased silent information regulator 1 (Sirt1) expression to reduce the phosphorylation of the mammalian target of rapamycin and its downstream effectors P70S6K and 4EBP1. Moreover, the induction of Sirt1 by TGC was inhibited by the specific inhibitor EX527. In the presence of EX527, TGC-induced autophagyspecific proteins were downregulated, while apoptotic and inflammatory factors were upregulated. In summary, our results demonstrate that TGC improved cardiac remodeling in a murine model of $\mathrm{Ml}$ by preventing cardiomyocyte inflammation and apoptosis but enhancing autophagy through Sirt1 activation.

\footnotetext{
Keywords: cardiac remodeling, Tongguan capsules, autophagy, apoptosis, Sirt1
} 


\section{INTRODUCTION}

Recent advances in the treatment of myocardial infarction (MI), such as early coronary reperfusion therapy and established standards medical care, have contributed to increased preservation of a viable myocardium and notably reduced the mortality rates (Cuenin et al., 2017). However, the parallel increase of the prevalence of and mortality from pathological myocardial remodeling and heart failure following MI has emerged as a growing challenging public health problem (Sharp et al., 2017). Currently, therapeutic strategies to prevent adverse left ventricular (LV) remodeling and heart failure after MI remain limited (Hassell et al., 2017). Thus, the attenuation of cardiac remodeling has long been the focus for efficient strategies to improve patients' post-infarct prognosis.

Studies on cardiomyocytes apoptosis and autophagy in the pathophysiological process of post-infarct adverse cardiac remodeling may provide novel treatment or prevention strategies (Kunapuli et al., 2006). Following MI, apoptosis occurs early in the remote myocardium and has been generally considered a potent inducer contributing to myocardial remodeling (Bharti et al., 2015). However, recent evidence has depicted an indefinite role of autophagy which plays an important part in maintaining intracellular hemostasis and function by degrading and recycling non-functional protein aggregates and scavenging damaged organelles at the basal levels (Levine and Klionsky, 2004). In addition, autophagy can be further induced in the heart by stress, such as acute and chronic ischemia, heart failure, and aging (Thapalia et al., 2014; Riquelme et al., 2016; Shirakabe et al., 2016). Cardiac specific loss of autophagy through a genetic deficiency of autophagy-related 5 (Atg5) was shown to cause cardiomyopathy, LV dilatation, and contractile dysfunction (Nakai et al., 2007). This is in line with another chronic cardiac ischemic pig model, which showed that repetitive myocardial stunning induced autophagy with less apoptosis followed by fully recovered heart function. These data suggest that autophagy may be essential for survival of the hibernating myocardium (Yan et al., 2005). On the contrary, inhibition of autophagy with 3-methyladenine (3MA) reduced autophagosome formation and subsequent autophagic cell death of rat cardiomyocyte-derived H9c2 cells during glucose deprivation (Aki et al., 2003). However, whether it is necessary for the survival of or detrimental to cardiomyocytes, the precise role of autophagy in pathological remodeling remains to be elucidated.

From the perspective of traditional Chinese medicine (TCM), the deleterious outcomes of coronary heart diseases are generally caused by $Q i$ (energy) deficiency and obstruction of the coronary circulation (Hou et al., 2014). Therefore, several Chinese herbal remedies with the effects of nourishing $Q i$ and activating blood circulation have been widely utilized in ischemic heart disease, including angina pectoris, MI, and heart failure (Mao et al., 2015, 2016b; Li X.Q. et al., 2016). Tongguan capsules (TGC) have received more attention due to their certified cardioprotective effects in human and animal models (Chen et al., 2012; Qi et al., 2013; Ma et al., 2015). Our previous data showed that TGC could markedly decrease cardiac injury biomarkers that led to improved cardiac function in rats with experimental MI (Chen et al., 2012). Most recently, in preclinical myocardial ischemia/reperfusion injury models, the administration of TGC prevented the occurrence of arrhythmias and limited infarction size (Qi et al., 2013). Importantly, the clinical application of TGC had a remarkable therapeutic effect on patients with coronary heart disease, which was evident in the regression of coronary atherosclerosis assessed by coronary computed tomography scans (Ma et al., 2015). These desirable pharmacological effects may be ascribed to the active components of this compound, mainly consisting of Salvia miltiorrhiza, Hirudo medicinalis, and Astragalus membranaceus. Based on the results of high-performance liquid chromatography fingerprint analysis, this patented medicine has been identified in nearly 15 active chemical components, including salvianolic acid B, fermlononetin, tanshinone I, tanshinone IIA, cryptotanshinone, and salvianolic acid A, most of which are established for cardioprotective therapies (Figure 1) (Pan et al., 2011; Zhang et al., 2011; Yuan et al., 2014; Lin et al., 2016; Oche et al., 2016). Inspired by the supportive evidence, we conducted this study to assess the efficacy of TGC on the pathological myocardial remodeling in mice subjected to MI. We demonstrated improved cardiac function and attenuated pathological adverse myocardial remodeling in post-infarct mice treated with TGC. Mechanistically, the protective role of TGC was largely a consequence of its inhibition of apoptosis and provocation of autophagy through silent information regulator 1 (Sirt1).

\section{MATERIALS AND METHODS}

\section{Experimental Design}

The experimental procedures were performed according to the Guide for the Care and Use of Laboratory Animals published by the US National Academy of Sciences (8th edition, Washington DC, 2011). The Institutional Animal Care and Use Committee of Guangdong Province Hospital of Chinese Medicine at Guangzhou University of Chinese Medicine approved the experimental animal protocol. All reagents were obtained from Sigma-Aldrich Chemicals (St. Louis, MO, United States) unless otherwise specified.

After thoracotomy surgery, mice were randomized into six groups: sham operated mice given vehicle (saline) or TGC; MI mice given vehicle or TGC; MI mice given TGC plus 3MA (autophagy inhibitor); and MI mice given TGC plus EX527 (Sirt1 inhibitor). TGC (batch number 161201; Guangdong Province Hospital, Guangzhou,) was dissolved in $0.9 \%$ normal saline and delivered as a daily oral gavage at a dose of $5 \mathrm{mg} / \mathrm{kg}$ for 6 weeks. Previous data demonstrated its effect and safety (Qi et al., 2013). The 3MA was administered by intraperitoneal injection ( $15 \mathrm{mg} / \mathrm{kg}$, twice per week) for 6 weeks. EX527 was intraperitoneally injected at the dose of $5 \mathrm{mg} / \mathrm{kg}$ twice per week for 6 weeks. These doses of 3MA and EX527 were previously reported not to cause apparent adverse effects in the rodent 


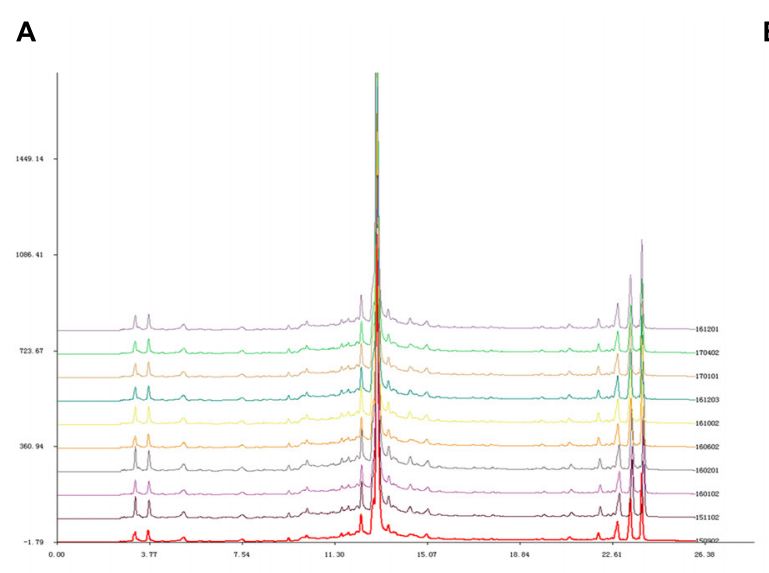

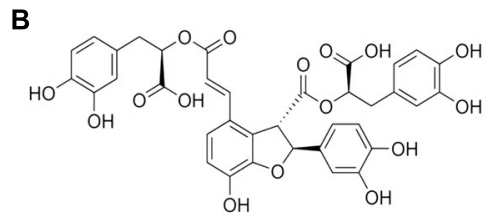

salvianolic acid $B$

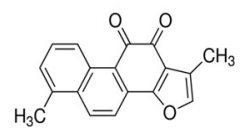

tanshinone I

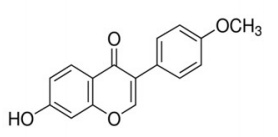

formononetin

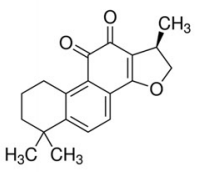

cryptotanshinone

FIGURE 1 | High-performance liquid chromatography fingerprint analysis of Tongguan capsules. (A) HPLC analysis identified nearly 15 active chemical components, including salvianolic acid B, fermlononetin, tanshinone I, cryptotanshinone and tanshinone IIA. The similarity of 10 batches of TGC and the reference fingerprint was greater than $99.72 \%$. (B) Structure of representative components.

heart (Wu et al., 2014; Sin et al., 2015). After 6 weeks, the surviving mice were subjected to echocardiographic assessment; subsequently, the hearts were extracted for histology, enzymelinked immunosorbent assay (ELISA), western blotting or other analysis.

\section{Mouse Model of MI}

Three-month-old male C57BL/6 mice were obtained from the Experimental Animal Center of Guangdong Province (Guangzhou, China). After being weighed, the mice were anesthetized, intubated, and mechanically ventilated using a rodent ventilator. The hearts were exposed by thoracotomy and subsequent pericardiotomy. MI was induced by permanent left anterior descending coronary artery ligation using 8-0 silk suture, and then confirmed by ST segment elevation or the newly emerging pathological Q waves on an electrocardiogram using the BL-420S Biological Function Experimental System (TME, Shanghai, China). Sham-operated animals underwent the same procedure without ligation of the left coronary artery.

\section{Transthoracic Echocardiography}

Cardiac LV function and structure were non-invasively examined with transthoracic echocardiography via the Vevo 770 highresolution imaging system (Visual Sonics, Toronto, Canada) using two-dimensional guided M-mode echocardiography, a 15-MHz RMV-707B scanning head before and 3 days and 6 weeks after coronary ligation. All mice were anesthetized using $2 \%$ isoflurane/oxygen inhalation before the echocardiography examination. The M-mode measurements were acquired at the level of the papillary muscles with the cursor positioned perpendicular to the interventricular septum and posterior wall. LV dimensions including LV end diastolic dimension (LVEDD) and LV end systolic dimension (LVESD) were averaged from more than 10 cardiac cycles according to the Guidelines of the American Society of Echocardiography (Manning et al., 1994).

\section{Cardiac Histology}

Six weeks after the coronary ligation, the mice were anesthetized and their hearts were harvested, rinsed in normal saline, and surface-dried. The LV myocardium was weighed after removal of the atrial and right ventricular myocardium. The heart mass index (heart mass/body mass in milligrams per gram) were calculated. Subsequently, the ventricular myocardium was flash-frozen in liquid nitrogen and stored at $80^{\circ} \mathrm{C}$ until used in molecular analyses or fixed in specific fixatives for the histological analyses. The entire procedure was performed in cold conditions.

The murine heart specimens were fixed in $4 \%$ formaldehyde overnight and embedded in paraffin. After deparaffinization and rehydratation, $4-\mu \mathrm{m}$-thick sections were prepared, mounted on glass slides, and stained with hematoxylin and eosin or Masson's trichrome staining. Cardiac scar circumference and fibrosis were quantitatively analyzed using a microscope at $40 \times$ magnification and a color image analyzer (QWinColour Binary 1; Kirkland, LEICA).

\section{Terminal Deoxynucleotidyl Transferase dUTP Nick End Labeling Staining}

The DNA fragmentations were determined using a FluoresceinFragEL kit (Oncogene Research Products, Boston, MA, United States) according to the manufacturer's instructions. Terminal deoxynucleotidyl transferase dUTP nick end labeling (TUNEL) staining was visualized using specific green fluorescence and nuclei by $4^{\prime}$-6-diamidino-2-phenylindole (DAPI). The number of TUNEL-positive cardiomyocytes nuclei was counted and normalized per total nuclei identified by DAPI staining in the same sections using NIS-Element imaging software (Nikon Instruments, Tokyo, Japan).

\section{Immunofluorescence}

After deparaffinization, the sections were incubated with a primary antibody against microtubule-associated LC3 (MBL 
International, Woburn, MA, United States). To observe autophagic activity in the cardiomyocytes, sections immunostained with anti-LC3 followed by Alexa 568 (red; Molecular Probes, Sunnyvale, CA, United States) were also labeled with anti-myoglobin antibody (DAKO Japan, Kyoto, Japan) followed by Alexa 488 (green; Molecular Probes). These sections were then counterstained with Hoechst 33342 and examined under an Eclipse E1000 microscope and Nikon Digital Sight Camera (Nikon Instruments, Tokyo, Japan).

\section{Electron Microscopy}

Cardiac sample sections from different treatment groups were cut into 1-mm-thick slices and fixed with glutaraldehyde in sodium cacodylate buffer overnight at $37^{\circ} \mathrm{C}$. The specimens were washed in distilled water, stained with $2 \%$ osmium tetroxide and $0.5 \%$ uranyl acetate, dehydrated through a graded series of ethanol and propylene oxide, embedded in epoxy resin, and polymerized for 3 days. Ultrathin sections of $90 \mathrm{~nm}$ were cut and mounted on Formvar-coated slot copper grids. Images were acquired of the thin sections using a FEI Tecnai 12 transmission electron microscope (Hitachi H-600, Tokyo, Japan) equipped with a Veleta CCD digital camera (Olympus Soft Imaging Solutions GmbH, Münster, Germany). For quantification, 20 random regions for each sample were considered.

\section{Enzyme-Linked Immunosorbent Assay}

Levels of inflammatory cytokines including tumor necrosis factor (TNF) $-\alpha$, interleukin (IL)-1 $\beta$, and IL- 6 in the myocardium were measured using commercially available ELISA kits (BioTech, MN, United States). All spectrophotometric readings were obtained with a microplate reader according to the manufacturer's instructions (Multiskan MK3, Thermo Scientific, United States).

\section{Western Blotting}

Left ventricular samples were homogenized in ice-cold buffer containing $20 \mathrm{mM}$ Tris- $\mathrm{HCl}, 1 \mathrm{mM}$ EDTA, $5 \mathrm{mM}$ magnesium citrate, $3 \mathrm{mM}$ phenylmethylsulfonyl fluoride, $2 \mathrm{mM}$ sodium orthovanadate, $20 \mathrm{mg} / \mathrm{ml}$ aprotinin, and a protease inhibitor cocktail. The prepared samples were analyzed using western blotting as previously described (Mao et al., 2016a). Briefly, $30 \mu \mathrm{g}$ of protein was loaded into each lane, separated with $8-12 \%$ sodium dodecyl sulfate-polyacrylamide gel, and then transferred onto a polyvinylidene difluoride membrane. The membrane was probed first with primary antibodies [anti-GAPDH, anti-ANP, anti-caspase3, anti-Bcl2, anti-Bax, anti-LC3, anti-p62, anti-mammalian target of rapamycin (mTOR), and anti-Sirt1] overnight at $4^{\circ} \mathrm{C}$ and then with a horseradish peroxidase-conjugated goat anti-rabbit (1:3000) or goat anti-mouse (1:3000) IgG secondary antibody for $2 \mathrm{~h}$ at room temperature. The probed protein bands were visualized with enhanced chemiluminescence reaction (Amersham Life Sciences Inc., Marlborough, MA, United States) and were quantified using densitometry (Chemidoc, Biorad, United States). All experiments were separately repeated at least three times.

\section{Statistical Analysis}

Data are presented as the groups and were compared using Student's $t$-test or one-way analysis of variance using SPSS 12.0 software for Windows. The cumulative survival rate of the rats after MI was analyzed by the Kaplan-Meier method with a log-rank test. Values of $P<0.05$ were considered significant.

\section{RESULTS}

\section{TGC Improved Cardiac Function of Mice After Coronary Ligation}

During the first 2 days after coronary ligation, the vehicle and TGC groups had similar mortality rates. However, the overall survival rates of the vehicle group were considerably lower than those of TGC group at 42 days after coronary ligation (57.69\% vs. $82.14 \%, P<0.05)$ with a hazard ratio of 2.77 (95\% confidence interval, 1.11-7.57). Furthermore, no mice died in the sham groups treated with TGC or vehicle (Figure 2A).

Echocardiography was performed to assess the cardiac function of mice at 3 days and 6 weeks after coronary ligation. Mice from the TGC and vehicle groups showed notable cardiac dysfunctional at 3 days after surgery, without noticeable intergroup differences. However, compared to the vehicle group, the TGC group demonstrated reduced LVEDD and LVESD, improved percent ejection fraction (\%EF), and improved fractional shortening (\%FS) at 6 weeks after coronary ligation (Figures 2B,C and Supplementary Table S1).

Along with echocardiography, hemodynamic analysis suggested a higher systolic pressure in the TGC group than the vehicle group. In the TGC group, there was a trend toward a decreased heart rate compared to the vehicle group, although the statistical difference was not significant. Moreover, we found no significant difference in cardiac function between TGC- and vehicle-treated mice that received sham surgery, suggesting that TGC does not affect LV function under non-surgical conditions.

\section{TGC Ameliorate Ventricular Fibrosis in Mice After Coronary Ligation}

After MI, the heart undergoes structural remodeling, resulting in a more spherical shape. Isolated hearts from the TGC group demonstrated a less spherical shape than those from the vehicle group 6 weeks after MI, indicating mitigated global cardiac remodeling in mice treated with TGC (Figure 3A).

The most striking finding of our study was a marked difference in scar expansion. In vehicle-treated mice, the scar comprised a much larger percent circumference of LV than in the TGC group, indicating the post-MI scar expansion was attenuated by TGC (Figure 3B).

Interstitial fibrosis is the hallmark of cardiac remodeling (Mao et al., 2014). As shown in Figure 3C, fibrotic areas were reduced in the TGC heart, suggesting suppressed interstitial fibrosis in the TGC-treated heart.

Myocyte size were analyzed in the border and the remote zones (Figure 3D). Increased myofilament density and decreased myocyte size were observed in the border zone of the TGC 


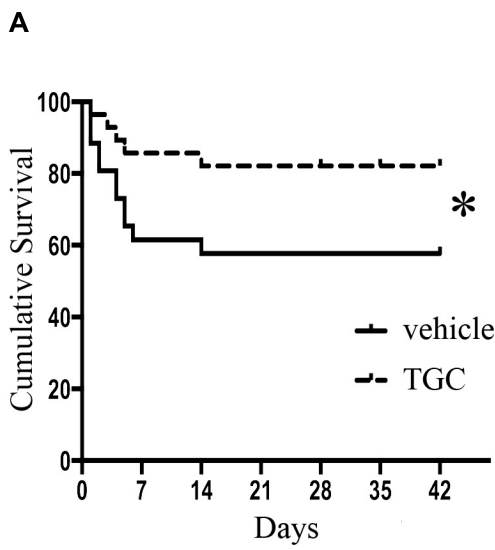

C
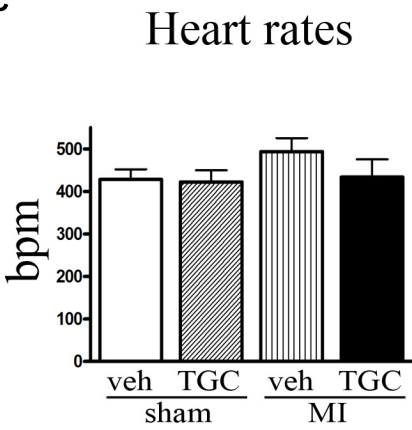

LVESD

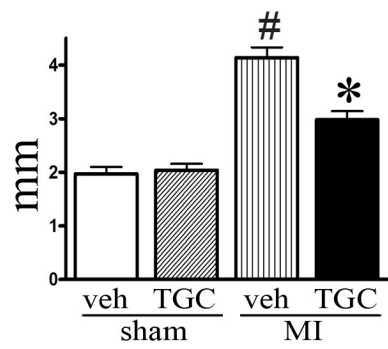

B

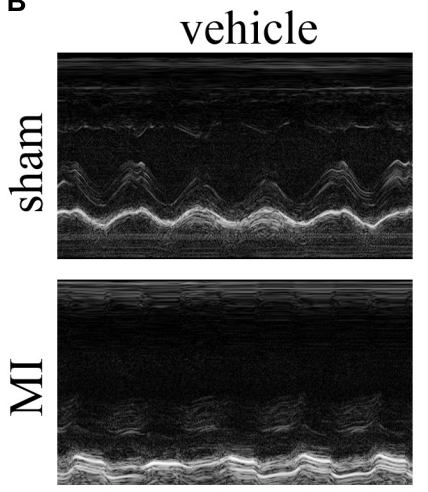

TGC

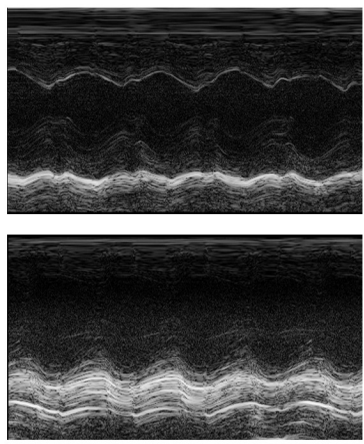

Systolic pressure

LVEDD
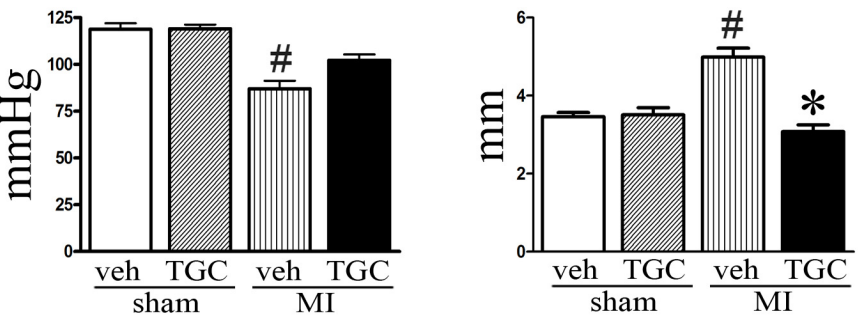

LVEF

LVFS
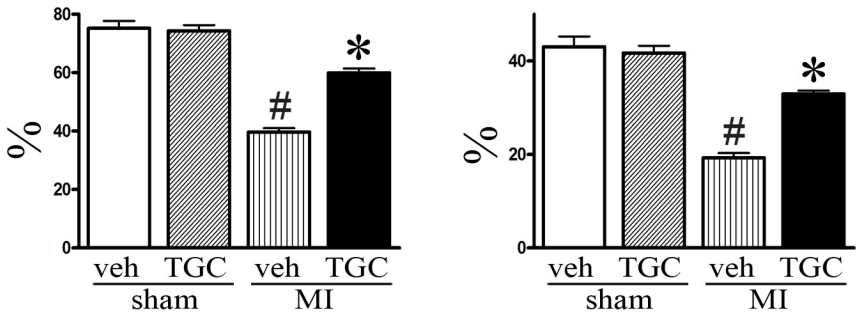

FIGURE 2 | TGC improved mice survival, cardiac morphology and function after MI. (A) Kaplan-Meier survival curve of MI mice treated with TGC ( $n$ = 28) or vehicle ( $n=26), \chi^{2}=3.94, P<0.05$. (B) Representative image of echocardiography of mice at 6 weeks after MI. (C) Quantitative analysis of LV ejection fraction (LVEF), fractional shortening (LVFS), left ventricular end-diastole diameter (LVEDD), LV end-systolic diameter (LVESD), heart rates, and systolic pressure. ${ }^{\#}<<0.05$ versus sham mice treated with vehicle; ${ }^{*} P<0.05$ versus MI mice treated with vehicle. Veh, vehicle; TGC, Tongguan capsules.

group compared to the vehicle group. In contrast, no significant difference in either myofilament density or myocyte size was observed in the remote zone between the two groups, suggesting that TGC attenuated myocyte hypertrophy and secondary cell loss in the border zone after MI.

It has been established that atrial natriuretic peptide (ANP) closely correlates with cardiac remodeling and dysfunction severity (Omland et al., 1996). Therefore, we assessed ANP expression in vehicle and TGC mice using western blotting analysis. As revealed in Figure 3E, ANP expression level was significantly downregulated in the TGC group compared to the vehicle group, suggesting the attenuation of deteriorated LV function in the TG group. Collectively, these results reveal that TGC treatment improved cardiac remodeling after MI.

\section{TGC Decreased Inflammatory Cytokine Expression in Mice Subjected to Coronary Ligation}

Myocardial ischemia initiates an inflammatory response characterized by an accumulation of leukocytes in the injured myocardium, while cytokines expression further promotes adverse LV remodeling and heart failure (Velagaleti et al., 2008). To elucidate the effect of TGC on post-infarct inflammation, 


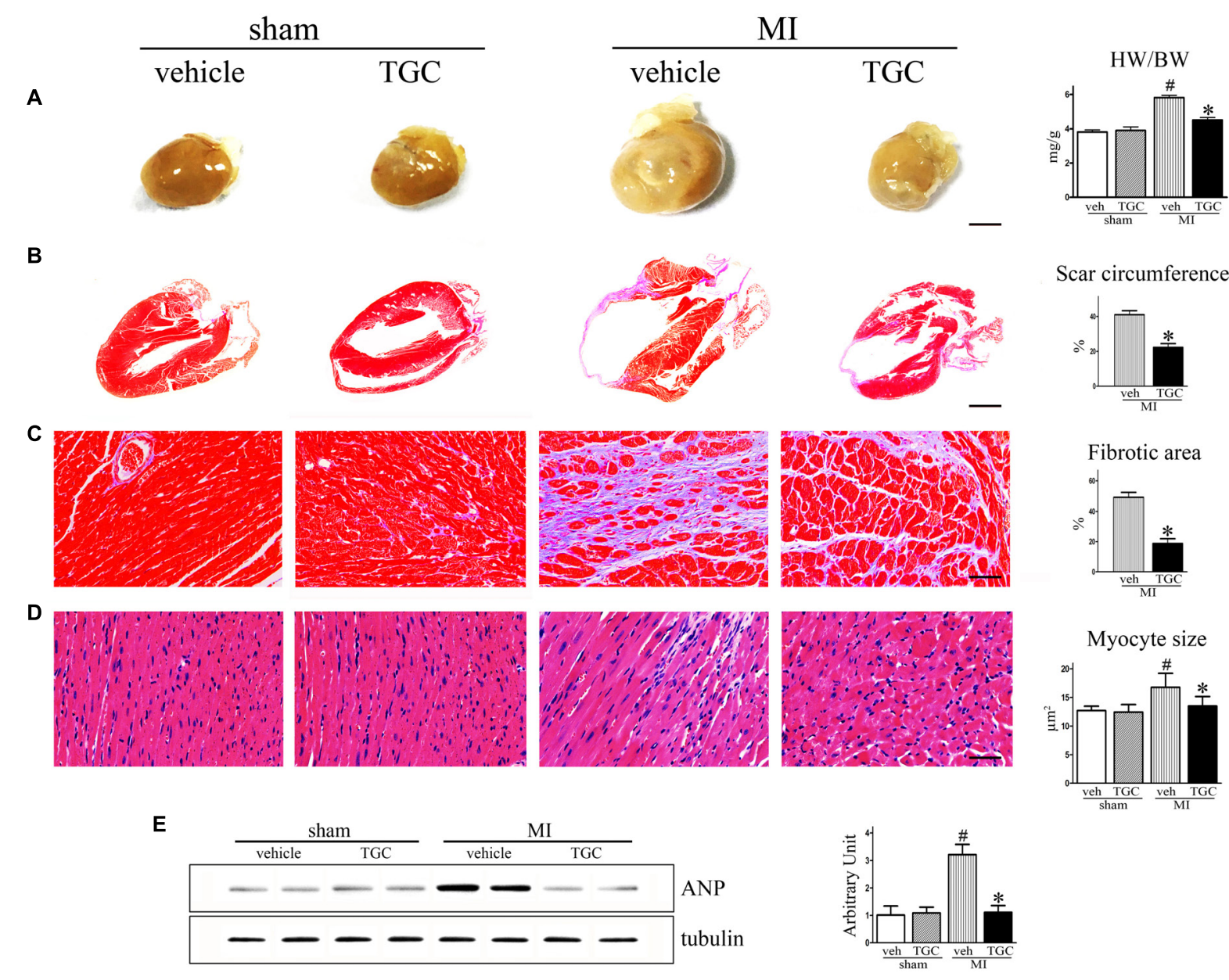

FIGURE 3 | TGC ameliorated ventricular fibrosis after MI. (A) Hearts from vehicle-treated mice appear more spherical relative to TGC-treated and sham mice, scale bar $=3 \mathrm{~mm}$. (B,C) Paraffin-embedded sections of myocardium were stained with Masson's trichrome showing the effects of TGC on scar circumference (scale bar $=5 \mathrm{~mm}$ ) and ventricular fibrosis (scale bar $=50 \mu \mathrm{m}$ ). (D) Representative image of hematoxylin and eosin showing myocyte size from mice treated with TGC or vehicle; bars $=50 \mu \mathrm{m}$. (E) Atrial natriuretic peptide expression in infracted hearts following TGC treatment was assessed by western blotting analysis. Graph right shows densitometric quantification. ${ }^{\#} P<0.05$ versus sham mice treated with vehicle; ${ }^{*} P<0.05$ versus Ml mice treated with vehicle. Veh, vehicle; TGC, Tongguan capsules.

we evaluated the expression of TNF- $\alpha$, IL- $1 \beta$ and IL- 6 in the myocardium using commercial ELISA kits. TGC dramatically decreased the expressions of TNF- $\alpha$, IL- $1 \beta$, and IL- 6 in the myocardium tissue compared to the vehicle group (Figure 4A).

In parallel, there was a remarkable decrease in immunedetectable antibodies against inflammatory factors TNF- $\alpha$, IL- $1 \beta$, and IL- 6 in the presence of TGC in the myocardium, which further supports results obtained from ELISA (Figure 4B).

\section{TGC Inhibited Apoptosis in Mice After Coronary Ligation}

Cardiomyocyte apoptosis contributes to post-infarct cardiac remodeling and subsequent cardiac dysfunction (Haudek et al., 2007). To identify whether TGC protects the post-infarct myocardium against apoptosis, we assessed apoptotic biomarkers expression using TUNEL staining and western blotting analysis. There were dramatically fewer apoptotic (TUNEL-positive) cells on the border of the MI hearts of the TGC group compared to the vehicle group (Figure 5A), suggesting that TGC antiapoptotic properties. This result was confirmed by measurement of the cleaved caspase 3, B cell lymphoma-2 (Bcl-2), and Bcl2 -associated $\mathrm{X}(\mathrm{Bax})$ via western blotting analysis. There was a significant increase in Bcl-2 and decrease in cleaved caspase 3 and Bax at the protein level on the border of the MI hearts after TGC treatment (Figure 5B).

\section{Upregulation of Autophagy by TGC in Mice After Coronary Ligation}

In supporting evidence, autophagy, associated with cardiomyocytes survival in ischemia, is involved in the regulation of LV remodeling; thus, we investigated whether TGC attenuated LV remodeling by regulating cell autophagy after MI.

The LC3-II to LC3-I ratio and Atg5 level were used to assess autophagy in the post-infarct myocardium, while the autophagy 
A

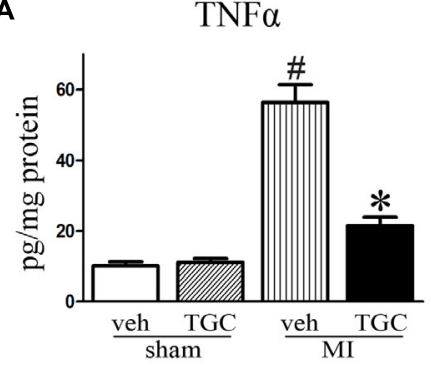

IL-1 $\beta$

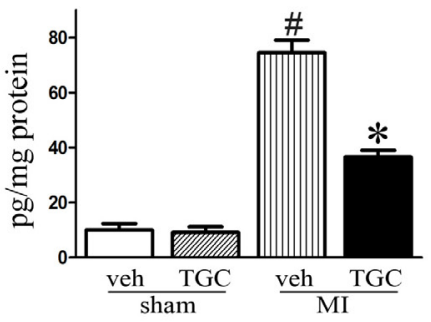

IL-6

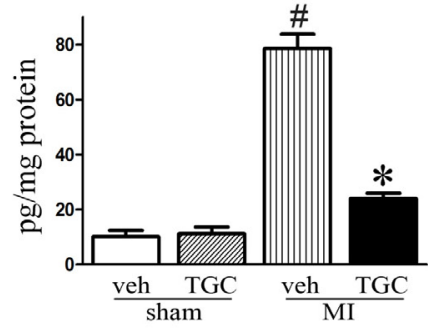

B

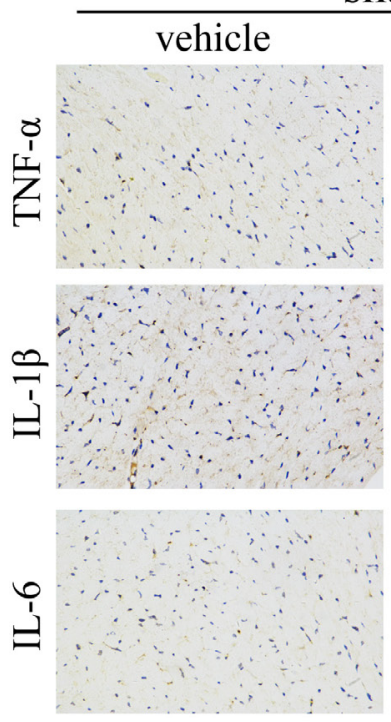

sham
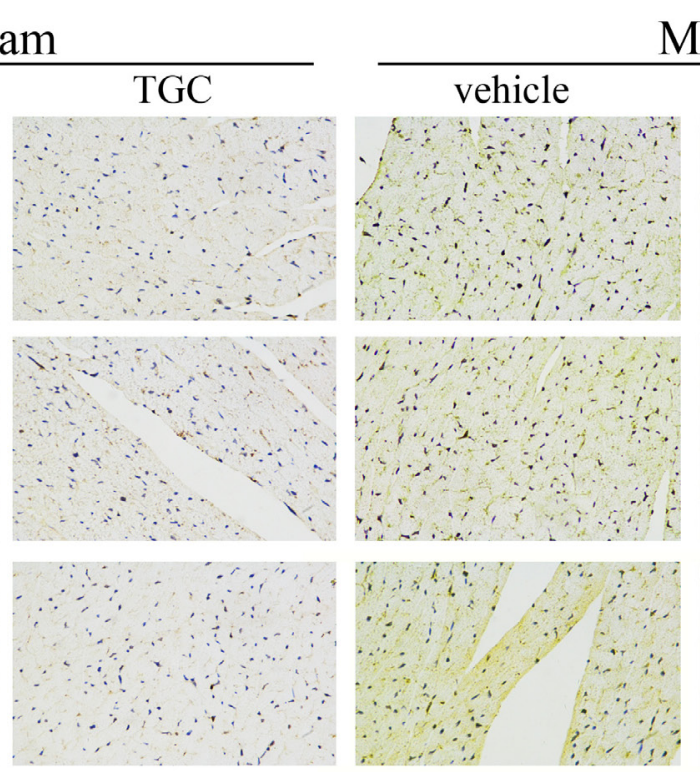

MI

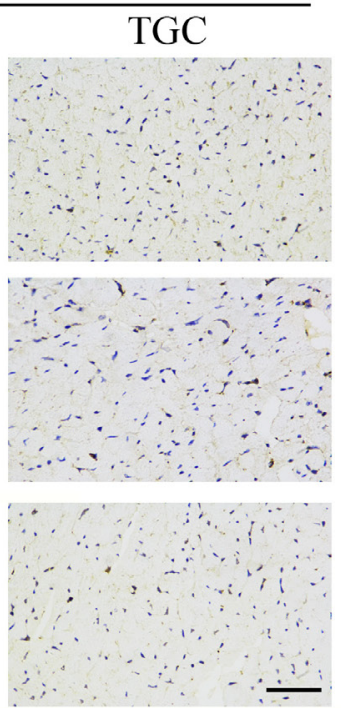

FIGURE 4 | TGC decreased inflammatory factors expression after MI. (A) The production of TNF- $\alpha$, IL-1 $\beta$, and IL-6 assessed by ELISA kits in the myocardium. (B) Immunohistochemical analysis of inflammatory factors expression in the infarct border zone of the heart (scale bar $=50 \mu \mathrm{m}$ ). " $P<0.05$ versus sham mice treated with vehicle; ${ }^{*} P<0.05$ versus Ml mice treated with vehicle. Veh, vehicle; TGC, Tongguan capsules.

substrate SQSTM1/p62 was associated with autophagic flux (Klionsky et al., 2016). There was an upregulation of the autophagic molecular marker, Atg5, in the TGC group compared to the vehicle group (Figure 6A). Furthermore, LC3-II to LC3I ratio was increased but the SQSTM1/p62 expression was decreased in the TGC-treated MI hearts, suggesting that TGC causes enhanced autophagy in cardiomyocytes in response to myocardial ischemia and infarction. The enhanced autophagy in TGC-treated MI hearts was further confirmed by the increased autophagic vacuoles on electron microscopy (Figure 6B) and LC3-positive punctate staining assessed by immunofluorescence (Figure 6C).

\section{Inhibition of Autophagy Attenuated TGC-Induced Anti-Remodeling Effect}

Considering that accumulating evidence suggests that autophagy plays dual roles in cytoprotection and cell death, we then evaluated the effects of autophagy in the TGC-induced antiremodeling effect in post-infarct hearts. As shown in Figure 7, combination treatment with autophagic inhibitor 3MA resulted in a significantly increased ratio of heart weight to body weight, dilatation of LV-internal dimension, scar circumference, fibrosis area, and decrease of LVEF or FS compared to TGC treatment alone. Furthermore, TUNEL staining also demonstrated that combination treatment of 3MA diminished TGC-induced antiapoptosis effects (Figure 7). Taken together, the results indicated that TGC-induced autophagy plays an important protective role against cardiac injury and apoptosis after MI.

\section{TGC Downregulated the mTOR/P70/S6K/4EBP1 Pathway in Mice After Coronary Ligation}

Existing data demonstrate that mTOR and its downstream effectors P70S6K and 4EBP1 are involved in autophagy after ischemia (Guo et al., 2012). Accordingly, we determined whether TGC enhanced autophagy in MI mice via regulation of the mTOR/P70/S6K/4EBP1 pathway in the MI heart. First, we examined the mTOR expression by western blot analysis. As shown in Figure 8, TGC markedly reduced the mTOR (Ser2448) phosphorylation levels. Next, we examined the phosphorylation state of P70S6K (Thr309) and 4EBP1 (Thr70), established targets 


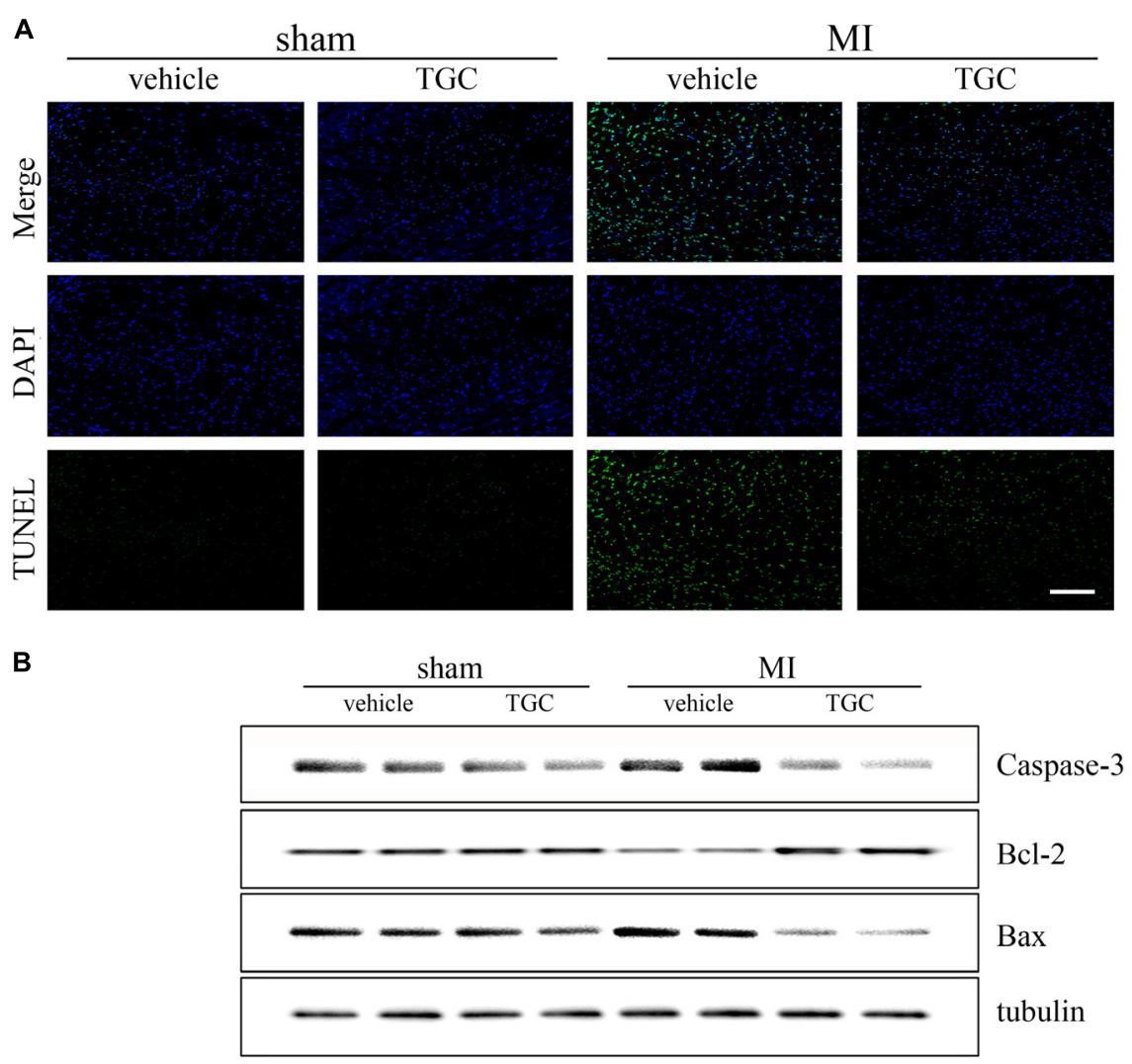

Caspase-3

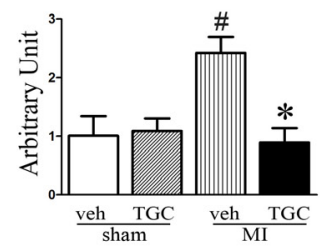

Bcl-2

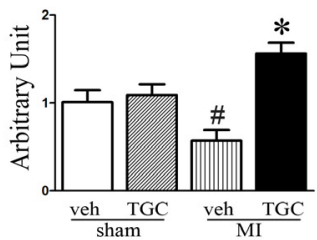

Bax

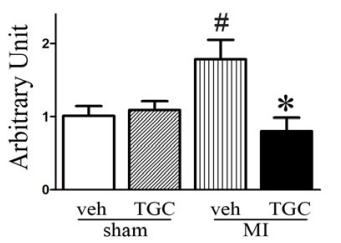

FIGURE 5 | TGC attenuated apoptosis after MI. (A) Apoptosis in myocardium from TGC or vehicle treated mice and sham group was evaluated by TUNEL assay. TUNEL-positive cells were significantly reduced in TGC group compared to the vehicle group, whereas few TUNEL-positive cells were detectable in the sham group (scale bar = $100 \mu \mathrm{m}$ ). DAPI nuclear staining in blue. (B) The caspase 3, Bcl-2, and Bax levels were assessed by western blotting analysis. Graph below shows densitometric quantification ( $n=4$ per group). ${ }^{\#} P<0.05$ versus sham mice treated with vehicle; ${ }^{*} P<0.05$ versus MI mice treated with vehicle. Veh, vehicle; TGC, Tongguan capsules.

of the mTOR1 complex. As shown in Figure 8, TGC also significantly decreased the phosphorylation levels of P70S6 kinase and 4EBP1. These results revealed that TGC is an effective inhibiter of the mTOR pathway in post-MI hearts.

\section{TGC Induced Autophagy and Decreased Apoptosis Through Sirt1 Activation}

It was previously reported that silent information regulator 1 (Sirt1) induced autophagy by inhibiting mTOR signaling (Salminen and Kaarniranta, 2009). For this reason, we next evaluated whether Sirt1 is involved in TGC-induced autophagy in mice with coronary ligation. As shown in Figure 9, TGC treatment indeed promoted Sirtl expression in post-infarct hearts compared to vehicle treatment.
As a NAD-dependent deacetylase, Sirt1 was demonstrated to regulate autophagy via its enzymatic activity (Lee et al., 2008). As expected, EX-527, a specific inhibitor of Sirt1 activity, attenuated TGC-induced autophagy as evidenced by decreased Atg5 levels and rescue of p62 and phosphorylated mTOR (Ser2448) levels (Figure 9B). Collectively, these results indicated that TGC-induced autophagy is mediated by Sirt1 activation and subsequently downregulates mTOR pathway mediators.

Moreover, the inhibition of Sirt1 activity by EX-527 increased forkhead box O1 (FOXO1) and Bax expressions and markedly decreased $\mathrm{Bcl} 2$ and $\mathrm{NF} \kappa \mathrm{B}$ expression compared to those in the TGC group (Figure 9B). These data suggest that TGC inhibits apoptosis and inflammation via Sirt1 as well. 


\section{A}
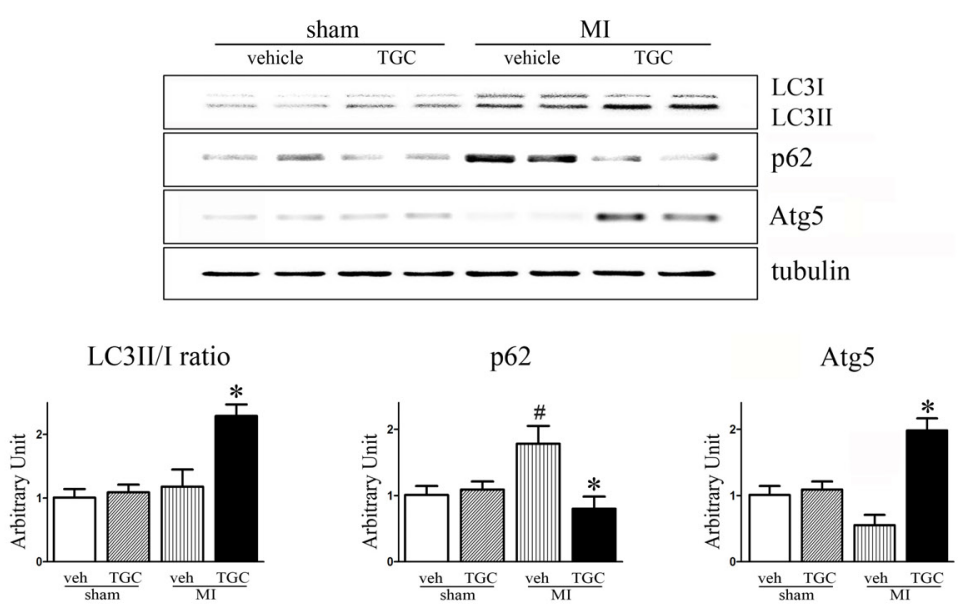

B

vehicle

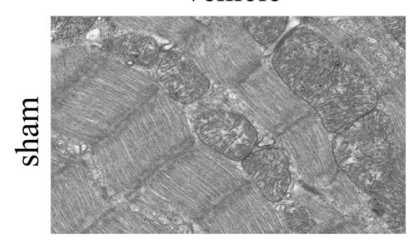

TGC
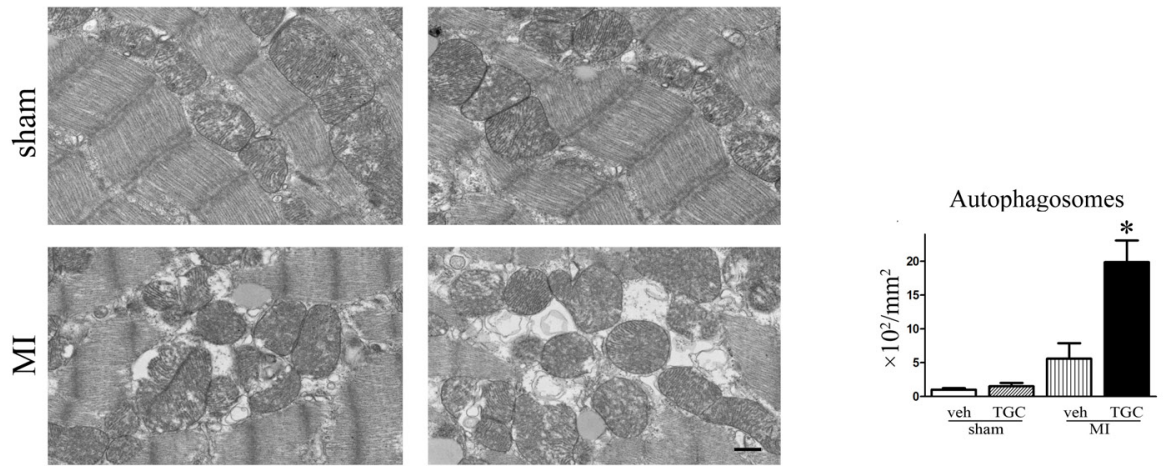

C

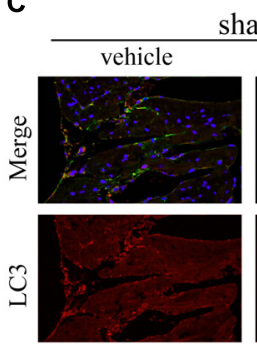

sham
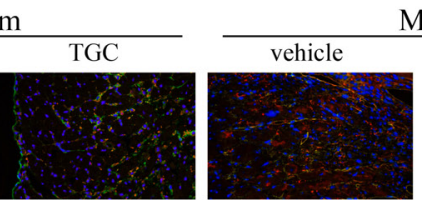

MI
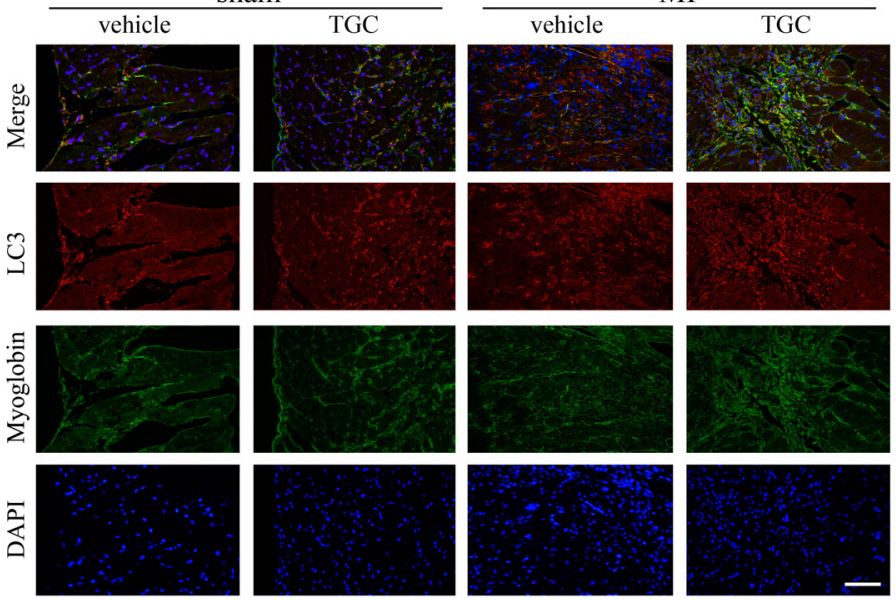

LC3 dots

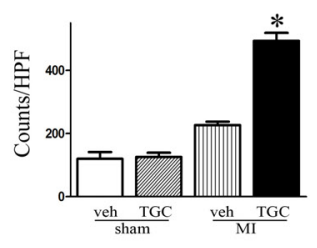

FIGURE 6 | TGC regulated autophagy after MI. (A) The expression of LC3II/LC3I, p62, and Atg5 in infracted heart was assessed by western blotting analysis.

(B) Electron micrographs show more autophagic vacuole formation in myocyte of Ml mice treated with TGC. Quantification of autophagy by measuring the average number of autophagic vacuoles per 10000* filed in electron microscope images; scale bars $=500 \mathrm{~nm}$. (C) Representative images of LC3 dots LV tissue sections of post-Ml heart; red, LC3; blue, DAPI-stained nuclei; green, myoglobin-positive cardiomyocytes; original magnification $\times 400$ (scale bar $=50 \mu \mathrm{m}$ ). ${ }^{\#} P<0.05$ versus sham mice treated with vehicle; $* P<0.05$ versus MI mice treated with vehicle. Veh, vehicle; TGC, Tongguan capsules.

\section{DISCUSSION}

Cardiac remodeling and dysfunction is a determinant of heart failure progression following MI. During the response to ischemic injury, in the ischemic and the remote non-infarcted myocardium, progressive LV remodeling occurs, which includes cardiomyocytes apoptosis, hypertrophy, and fibrosis (Bhatt et al., 2017). Developing strategies to reduce post-infarct cardiac 


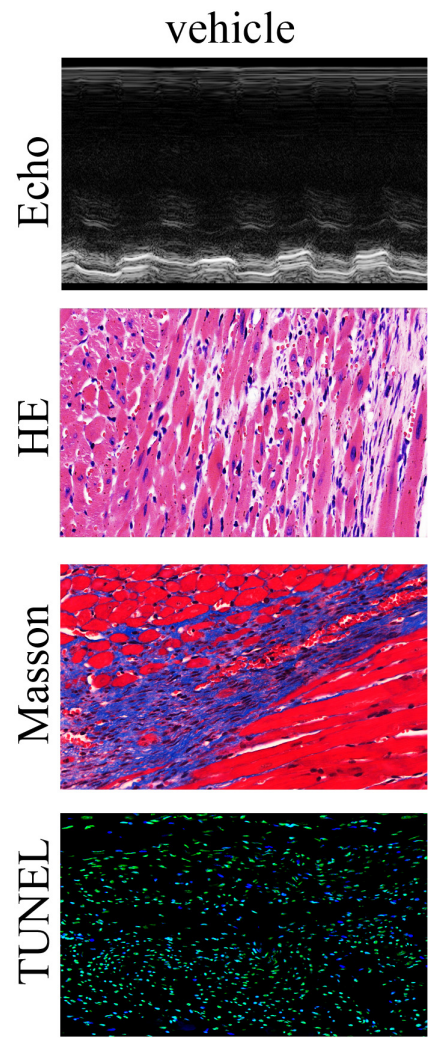

HW/BW

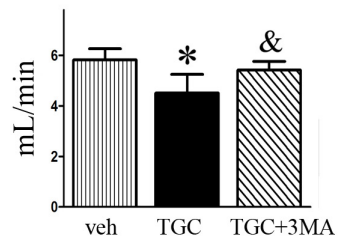

LVEF

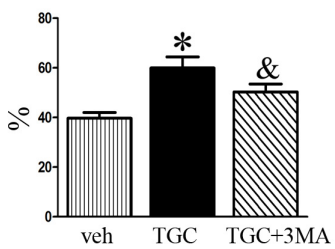

Scar circumference

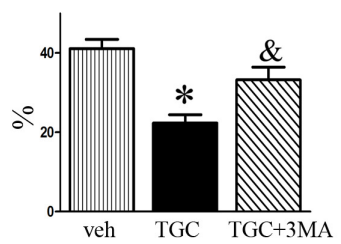

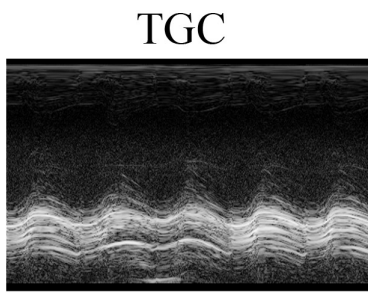
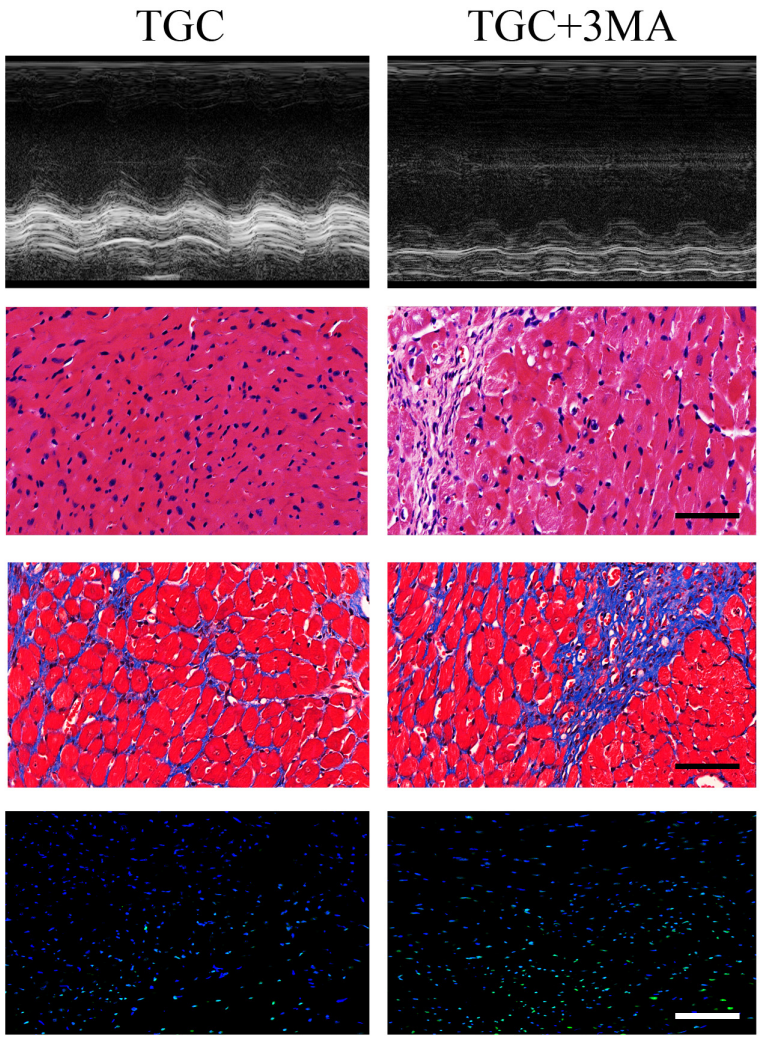

LVEDD

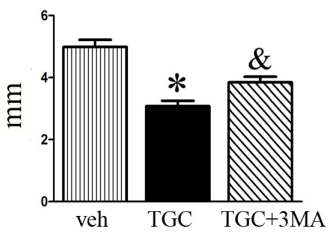

LVFS

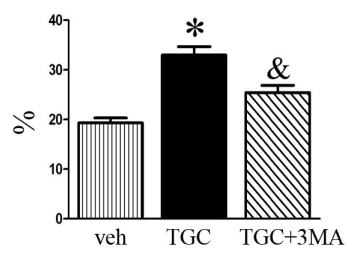

Fibrotic area

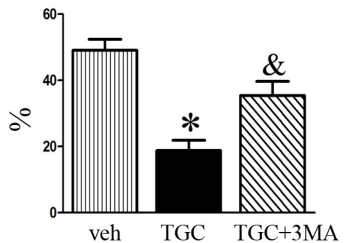

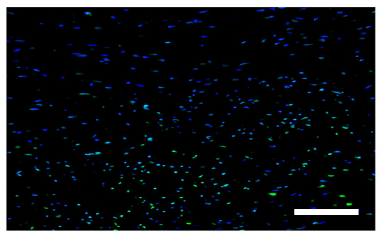

LVESD

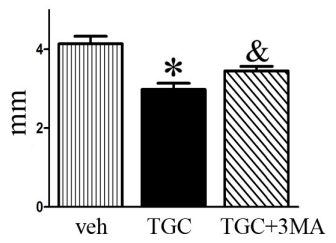

Myocyte size

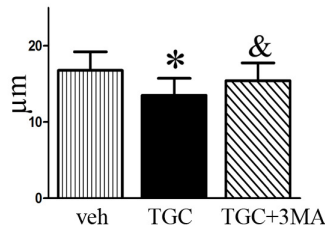

Apoptosis index

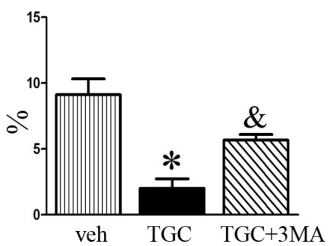

FIGURE 7 | Inhibition of TGC-induced autophagy enhanced LV remodeling after MI. MI mice were treated with TGC in the absence or presence of 3MA. Representative pictures of echocardiography, hematoxylin, and eosin (scale bar $=50 \mu \mathrm{m}$ ), Masson's trichrome (scale bar $=50 \mu \mathrm{m}$ ), and TUNEL (scale bar $=100 \mu \mathrm{m}$ ) stained LV tissue sections are shown in the upper panels. Quantitative analysis of heart weight to body weight ratio, left ventricular end-diastole diameter (LVEDD); LV end-systolic diameter (LVESD), LV ejection fraction (LVEF) and fractional shortening (LVFS), infarct size, fibrosis area, and apoptosis index are shown in lower panels. ${ }^{*} P<0.05$ versus MI mice treated with vehicle, ${ }^{\&} P<0.05$ versus MI mice treated with TGC. Veh, vehicle; TGC, Tongguan capsules. 

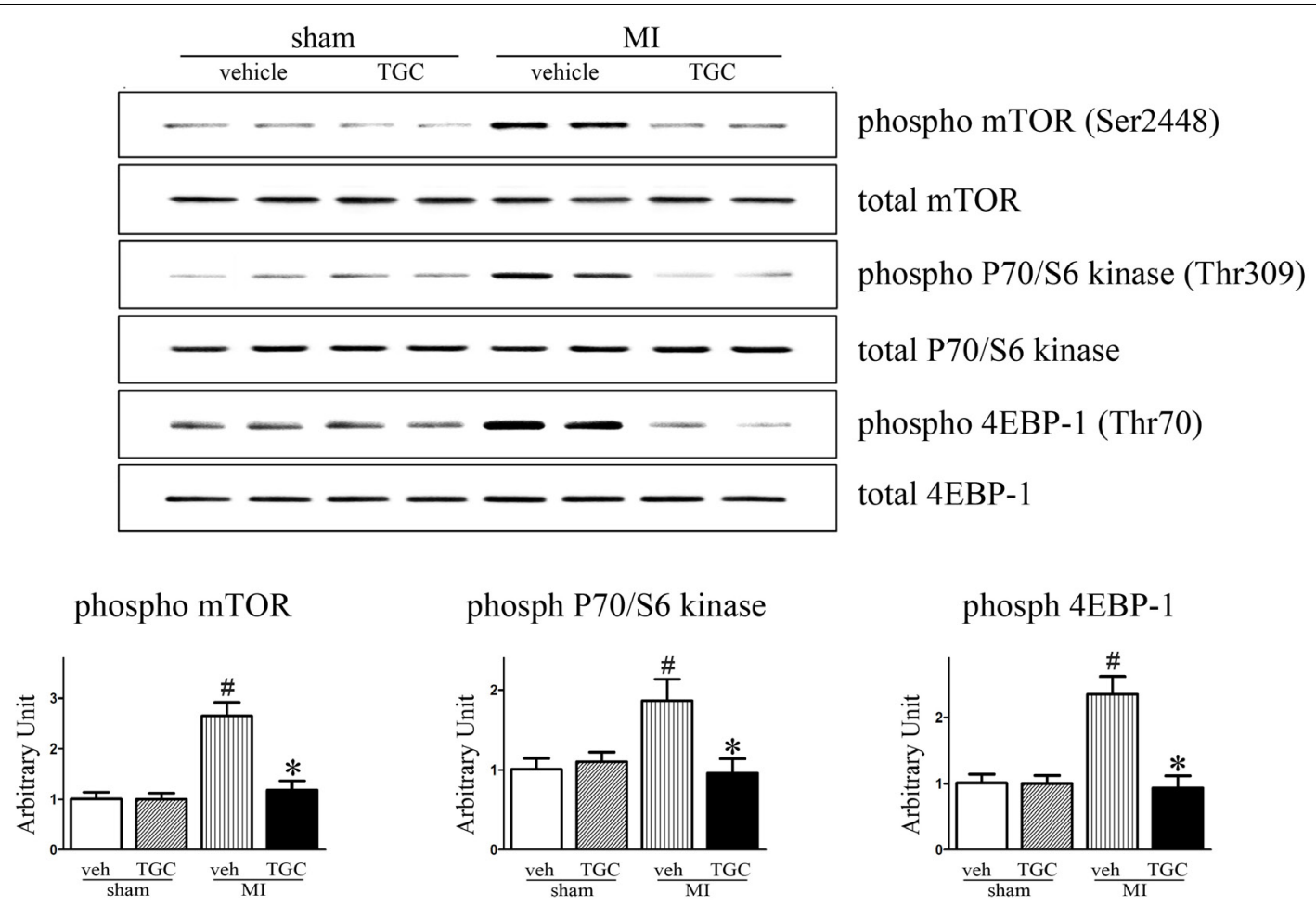

FIGURE 8 | TGC negatively regulated mTOR/P70/S6 kinase signaling pathway. Phosphorylation of mTOR (Ser2448) and other known downstream molecular targets including P70/S6- kinase (Thr309) and 4EBP1 (Thr70) in the hearts of MI mice treated with TGC or vehicle assessed by western blotting analysis. ${ }^{\#} P<0.05$ versus sham mice treated with vehicle; ${ }^{*} P<0.05$ versus MI mice treated with vehicle. Veh, vehicle; TGC, Tongguan capsules.

remodeling has become a major therapeutic challenge. In the present study, we confirmed a novel therapeutic approach to the treatment of post-MI remodeling using TCM. Our results suggest that TGC significantly attenuated pathological LV remodeling attributed to enhanced autophagy, reduced apoptosis and inflammation, and upregulated Sirt1 activation. Furthermore, a specific Sirtl inhibitor abolished the pro-autophagy, antiapoptotic, and anti-inflammatory effect of TGC as evidenced by the increased p62, Bax, and NFאB expressions and reduced Atg5 and Bcl-2 expressions. Thus, these results indicate that Sirtl activation by TGC contributes to the attenuated cardiac remodeling after $\mathrm{MI}$.

Sirt1 has drawn much attention for its ability to mediate longevity stimulated by resveratrol, a component of red wine seeds (Howitz et al., 2003). Importantly, Sirt1 is thought to play a crucial role in cardioprotection against myocardial ischemia/reperfusion injury through sumoylation which in turn induces lysine deacetylation of cytosolic proteins (Nadtochiy et al., 2011a). Sirt1 also mediates the protective effect by decreasing the transcriptional activity of NFKB through deacetylation of its subunit p65, which may in turn prevent inflammation and consequent cardiac remodeling (Nadtochiy et al., 2011b). Consistent with these findings, we found that TGC augmented Sirt1 expression as well as inhibited pro-inflammatory factors.

Several studies have shown that Sirt 1 activity inhibits apoptosis by deacetylating p53, a known apoptosis facilitator
(Yamamoto and Sadoshima, 2011). Sirt1 downregulation may contribute to rapid p53 activation and the consequent apoptotic death of cardiomyocytes during ischemia (Hsu et al., 2010). In addition, Sirt1 overexpression in transgenic mice induced deacetylation and nuclear translocation of FoxO1. This subsequently led to the upregulation Bcl-XL and Bcl2 upregulation and Bax downregulation (Hsu et al., 2010). This study also demonstrated that the specific inhibition of Sirt1 activity abolished TGC-induced anti-apoptotic effects. Accordingly, declined myocardial apoptosis via Sirtl is one mechanisms by which TGC attenuated post-MI cardiac remodeling. However, the precise mechanisms by which TGCactivated Sirtl controls gene expression through FoxO1 during the pathological remodeling process remain to be elucidated.

Autophagy is normally an important homeostatic mechanism that involves degradation and recycling of cytoplasmic components and organelles for maintaining myocyte function (Lum et al., 2005). In cardiac remodeling, an adaptive response protects cardiomyocytes against ischemic and hemodynamic stress by eliminating misfolded or unfavorably modified proteins and dysfunctional organelles that could disrupt cardiac function (Ceylan-Isik et al., 2013; Thomas et al., 2013). Recent studies have verified that Sirt1 activation could induce autophagy in cardiomyocytes and attenuate cardiac remodeling and contractile dysfunction (Zhang et al., 2014). In the absence of autophagy, the accumulation of polyubiquitinated proteins may be responsible for extended endoplasmic reticulum stress, resulting 


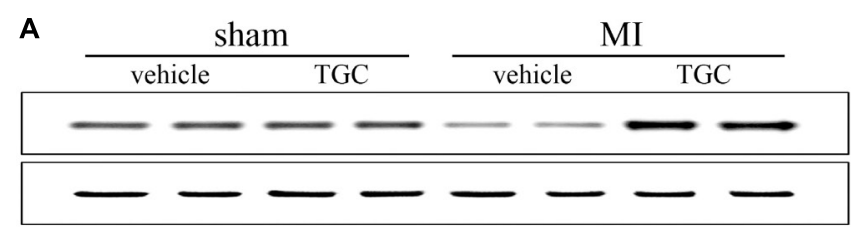

B

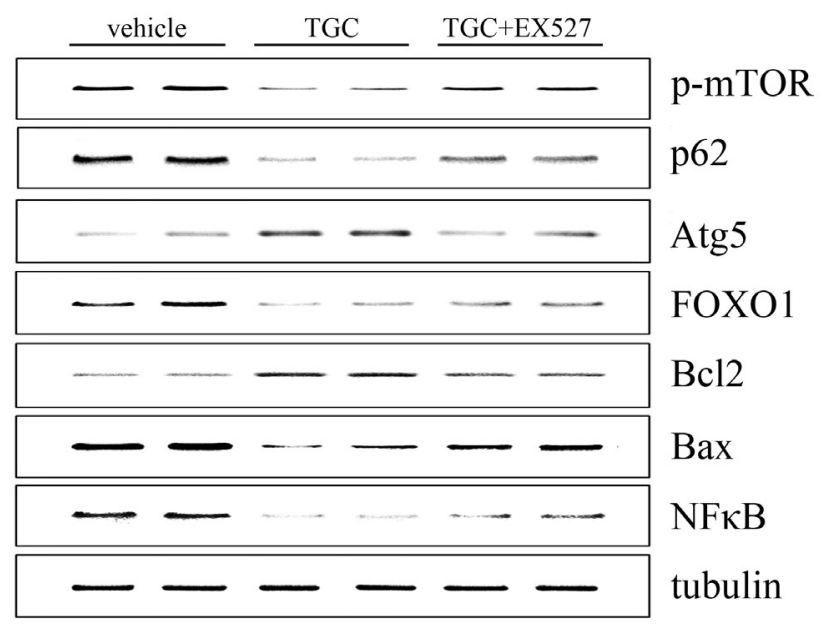

Sirt1 tubulin

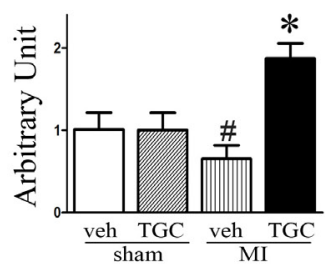

p-mTOR
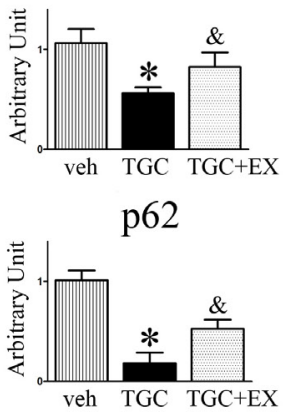

FOXO1

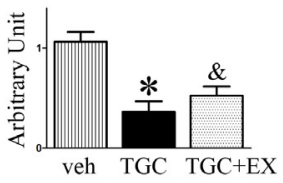

Bax

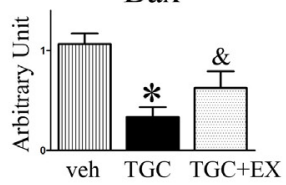

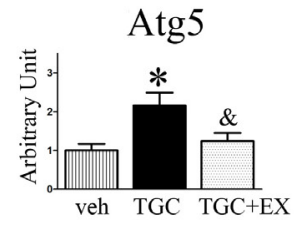

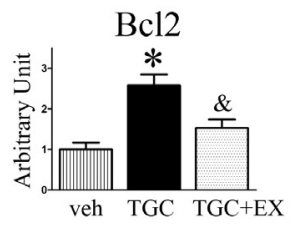

$\mathrm{NF} \kappa \mathrm{B}$

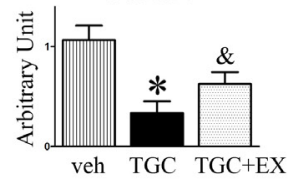

FIGURE 9 | Inhibition of Sirt1 suppressed TGC induced autophagy while increasing apoptosis in mice with MI. (A) Immunoblot analyses of Sirt1 in the heart homogenates of MI mice treated with TGC or vehicle. (B) MI mice were treated with TGC alone or TGC plus Sirt1 inhibitor EX527. The representative immunoblot analysis images of phosphorylation of mTOR (Ser2448), p62, Atg5, FOXO1, Bcl-2, Bax, and NFKB are shown. ${ }^{*} P<0.05$ versus Ml mice treated with vehicle, $\& P<0.05$ versus Ml mice treated with TGC. Veh, vehicle; TGC, Tongguan capsules.

in apoptosis (Nakai et al., 2007). Moreover, a previous study demonstrated that the increased cardiomyocyte autophagy acted as a cardioprotective response against apoptosis under a glucose deprivation condition through AMPK activation and mTOR inhibition (Matsui et al., 2007). Here we found that the LC3II/LC3-I ratio and autophagic vacuoles were significantly higher in TGC-treated mice than in vehicle-treated mice. However, the exact mechanism by which autophagy of cardiomyocytes protects against apoptosis in post-infarct hearts is unclear. Increasing evidence indicates that a potential mechanism is associated with the crosstalk between the autophagy initiation protein Beclin1 (BECN1) and anti-apoptotic protein Bcl-2, a switch between autophagy and apoptosis. Studies have demonstrated a strong link between Bcl-2 and BECN1 accompanied by the inhibition of autophagy in post-MI hearts with cardiac remodeling (Maejima et al., 2013). In contrast, disruption of Bcl-2 and BECN1 interaction gives rise to BECN1 being bound by the class III PtdIns3K to initiate autophagy (Maejima et al., 2016). In addition, phosphorylated Bcl-2 could stabilize the mitochondrial outer membrane and prevent pro-apoptotic proteins such as cytochrome $\mathrm{C}$ from escaping (or being released) into the cytoplasm (Yang et al., 1997). There are several other potential factors that regulate both autophagy and apoptosis, including Atgs, caspases, p53, and FADD-like IL-1 $\beta$-converting enzyme-inhibitory protein (FLIP) but further studies are needed to decipher the mechanism by which TGC regulates the switch between autophagy and apoptosis after MI (Li M. et al., 2016).

Autophagy has been demonstrated to play a dual role in cardiomyocyte survival. Both profitable and detrimental effects of autophagy have been described when cardiomyocytes were exposed to various stimuli (Gustafsson and Gottlieb, 2008). Therefore, it remains to be elucidated whether TGC-induced autophagy in the post-infarct myocardium is required for survival and thus was salutary or mediated the cell death and is thereby detrimental. In this study, the inhibition of TGCinduced autophagy by 3MA was accompanied by decreased cardiac dysfunction and cardiomyocyte survival, collectively suggesting that TGC-induced autophagy was protective in these 
processes. We deduced that the improved cardiac LV function was associated with enhanced autophagy, which facilitated animals to better respond to adverse ischemic stress (Matsui et al., 2007). The potential role of autophagy as a therapeutic target should be considered in future studies, while the mechanism of TGC-induced autophagy should be illustrated in detail.

The mTOR and its downstream signaling pathways are key regulators of autophagy and involved in pathological cardiac remodeling and even heart failure (Buss et al., 2009). However, the role of mTOR in ischemic heart disease remains undefined. Existing data shows that cardiac genetic mTOR overexpression was sufficient to provide substantial cardioprotection against ischemia-reperfusion injury both in vivo with transient coronary artery ligation and in Langendorffperfused hearts with transient global ischemia (Aoyagi et al., 2012). However, mTOR inhibition has also been shown to have definite protective effects when the myocardium suffers from ischemic or another stress stimulus by promoting autophagy (Shioi et al., 2003). Previous studies demonstrated that activated mTORC1 is associated with pathological cardiac remodeling in the heart (Ozcan et al., 2008). In contrast to Shioi's reports, we demonstrated here that TGC significantly inhibited the mTOR pathway, leading to increased autophagy in the context of myocardial ischemia. Considering that the mTOR plays a critical role in the cross-talk between autophagy and apoptosis, it is necessary to further investigate how the mTOR signaling pathway precisely regulates the switch between autophagy and apoptosis in TGC-treated cardiomyocytes (Eisenberg-Lerner et al., 2009).

In conclusion, our study demonstrated that TGC improved post-MI cardiac remodeling by affecting SIRT1 activity and subsequently enhancing autophagy, inhibiting apoptosis, and inflammation. These factors contribute to the cardioprotective role in post-MI. Therefore, TGC serves as a promising therapeutic candidate for the treatment for ischemic heart disease.

\section{REFERENCES}

Aki, T., Yamaguchi, K., Fujimiya, T., and Mizukami, Y. (2003). Phosphoinositide 3-kinase accelerates autophagic cell death during glucose deprivation in the rat cardiomyocyte-derived cell line H9c2. Oncogene 22, 8529-8535. doi: 10.1038/sj. onc. 1207197

Aoyagi, T., Kusakari, Y., Xiao, C. Y., Inouye, B. T., Takahashi, M., ScherrerCrosbie, M., et al. (2012). Cardiac mTOR protects the heart against ischemiareperfusion injury. Am. J. Physiol. Heart Circ. Physiol. 303, H75-H85. doi: 10.1152/ajpheart.00241.2012

Bharti, S., Rani, N., Bhatia, J., and Arya, D. S. (2015). 5-HT2B receptor blockade attenuates beta-adrenergic receptor-stimulated myocardial remodeling in rats via inhibiting apoptosis: role of MAPKs and HSPs. Apoptosis 20, 455-465. doi: 10.1007/s10495-014-1083-z

Bhatt, A. S., Ambrosy, A. P., and Velazquez, E. J. (2017). Adverse remodeling and reverse remodeling after myocardial infarction. Curr. Cardiol. Rep. 19:71. doi: 10.1007/s11886-017-0876-4

Buss, S. J., Muenz, S., Riffel, J. H., Malekar, P., Hagenmueller, M., Weiss, C. S., et al. (2009). Beneficial effects of Mammalian target of rapamycin inhibition on left ventricular remodeling after myocardial infarction. J. Am. Coll. Cardiol. 54, 2435-2446. doi: 10.1016/j.jacc.2009. 08.031

\section{AUTHOR CONTRIBUTIONS}

SM drafted this manuscript. SM, PC, and TL performed the experiments. LG did the statistical analysis. MZ critically revised the manuscript and contributed to the rationalization of the study. All authors read and approved the final manuscript.

\section{FUNDING}

This work was financially supported by the National Science Foundation (No. 81703877\&81703848), the National Basic Research Program of China (973 Program, 2015CB554400), the Science Foundation of Guangdong Province (No. 2015A030310437, 2015A030306049, and 2017A030310123), the Guangdong Provincial High-level University Construction Fund (A1-AFD018171Z11100), the Medical Science and Technology Research Foundation of Guangdong Province (A2016192), and the Chinese Medicine Bureau of Guangdong Province (A20162057). The sponsors have had no role in the project development, the collection of data, the preparation of this manuscript, or the decision to publish.

\section{ACKNOWLEDGMENTS}

The authors gratefully acknowledge the contributions of Dr. Shalina Taylor from Stanford University for a critical reading of the manuscript.

\section{SUPPLEMENTARY MATERIAL}

The Supplementary Material for this article can be found online at: https://www.frontiersin.org/articles/10.3389/fphys. 2018.00589/full\#supplementary-material

Ceylan-Isik, A. F., Dong, M., Zhang, Y., Dong, F., Turdi, S., Nair, S., et al. (2013). Cardiomyocyte-specific deletion of endothelin receptor A rescues aging-associated cardiac hypertrophy and contractile dysfunction: role of autophagy. Basic Res. Cardiol. 108:335. doi: 10.1007/s00395-0130335-3

Chen, Q. F., Zhang, M. Z., and Yang, C. (2012). [Experimental study of the myocardial protection on septic rats by tongguan capsule]. Zhongguo Zhong Xi Yi Jie He Za Zhi. 32, 1253-1257.

Cuenin, L., Lamoureux, S., Schaaf, M., Bochaton, T., Monassier, J. P., Claeys, M. J., et al. (2017). Incidence and significance of spontaneous ST segment re-elevation after reperfused anterior acute myocardial infarction- relationship with infarct size, adverse remodeling, and events at 1 year. Circ. J. 82, 1379-1386. doi: 10.1253/circj.CJ-17-0671

Eisenberg-Lerner, A., Bialik, S., Simon, H. U., and Kimchi, A. (2009). Life and death partners: apoptosis, autophagy and the cross-talk between them. Cell Death Differ. 16, 966-975. doi: 10.1038/cdd.2009.33

Guo, R., Hu, N., Kandadi, M. R., and Ren, J. (2012). Facilitated ethanol metabolism promotes cardiomyocyte contractile dysfunction through autophagy in murine hearts. Autophagy 8, 593-608. doi: 10.4161/auto.18997

Gustafsson, A. B., and Gottlieb, R. A. (2008). Recycle or die: the role of autophagy in cardioprotection. J. Mol. Cell. Cardiol. 44, 654-661. doi: 10.1016/j.yjmcc.2008. 01.010 
Hassell, M. E., Vlastra, W., Robbers, L., Hirsch, A., Nijveldt, R., Tijssen, J. G., et al. (2017). Long-term left ventricular remodelling after revascularisation for ST-segment elevation myocardial infarction as assessed by cardiac magnetic resonance imaging. Open Heart 4:e000569. doi: 10.1136/openhrt-2016-000569

Haudek, S. B., Taffet, G. E., Schneider, M. D., and Mann, D. L. (2007). TNF provokes cardiomyocyte apoptosis and cardiac remodeling through activation of multiple cell death pathways. J. Clin. Invest. 117, 2692-2701. doi: 10.1172/JCI29134

Hou, J., Wang, J., Lin, C., Fu, J., Ren, J., Li, L., et al. (2014). Circulating microRNA profiles differ between Qi-stagnation and Qi-deficiency in coronary heart disease patients with blood stasis syndrome. Evid. Based Complement. Alternat. Med. 2014:926962. doi: 10.1155/2014/926962

Howitz, K. T., Bitterman, K. J., Cohen, H. Y., Lamming, D. W., Lavu, S., Wood, J. G., et al. (2003). Small molecule activators of sirtuins extend Saccharomyces cerevisiae lifespan. Nature 425, 191-196. doi: 10.1038/nature01960

Hsu, C. P., Zhai, P., Yamamoto, T., Maejima, Y., Matsushima, S., Hariharan, N., et al. (2010). Silent information regulator 1 protects the heart from ischemia/reperfusion. Circulation 122, 2170-2182. doi: 10.1161/CIRCULATIONAHA.110.958033

Klionsky, D. J., Abdelmohsen, K., Abe, A., Abedin, M. J., Abeliovich, H., Acevedo Arozena, A., et al. (2016). Guidelines for the use and interpretation of assays for monitoring autophagy (3rd edition). Autophagy 12, 1-222. doi: 10.1080/ 15548627.2015.1100356

Kunapuli, S., Rosanio, S., and Schwarz, E. R. (2006). "How do cardiomyocytes die?" apoptosis and autophagic cell death in cardiac myocytes. J. Card. Fail. 12, 381-391. doi: 10.1016/j.cardfail.2006.02.002

Lee, I. H., Cao, L., Mostoslavsky, R., Lombard, D. B., Liu, J., Bruns, N. E., et al. (2008). A role for the NAD-dependent deacetylase Sirt1 in the regulation of autophagy. Proc. Natl. Acad. Sci. U.S.A. 105, 3374-3379. doi: 10.1073/pnas. 0712145105

Levine, B., and Klionsky, D. J. (2004). Development by self-digestion: molecular mechanisms and biological functions of autophagy. Dev. Cell 6, 463-477. doi: 10.1016/S1534-5807(04)00099-1

Li, M., Gao, P., and Zhang, J. (2016). Crosstalk between autophagy and apoptosis: potential and emerging therapeutic targets for cardiac diseases. Int. J. Mol. Sci. 17:332. doi: 10.3390/ijms17030332

Li, X. Q., He, J. C., Huang, P. X., and Cao, X. B. (2016). Chinese medicine syndromes in congestive heart failure: a literature study and retrospective analysis of clinical cases. Chin. J. Integr. Med. 22, 738-744. doi: 10.1007/s11655015-2085-6

Lin, C., Liu, Z., Lu, Y., Yao, Y., Zhang, Y., Ma, Z., et al. (2016). Cardioprotective effect of Salvianolic acid B on acute myocardial infarction by promoting autophagy and neovascularization and inhibiting apoptosis. J. Pharm. Pharmacol. 68, 941-952. doi: 10.1111/jphp.12567

Lum, J. J., DeBerardinis, R. J., and Thompson, C. B. (2005). Autophagy in metazoans: cell survival in the land of plenty. Nat. Rev. Mol. Cell Biol. 6, 439-448. doi: 10.1038/nrm1660

Ma, H., Wang, L., Huang, D., Liu, G., and Zhang, M. (2015). Tongguan capsule ameliorates coronary artery stenosis in a 40 -year-old woman. Drug Des. Dev. Ther. 9, 4413-4416. doi: 10.2147/DDDT.S85571

Maejima, Y., Isobe, M., and Sadoshima, J. (2016). Regulation of autophagy by Beclin 1 in the heart. J. Mol. Cell. Cardiol. 95, 19-25. doi: 10.1016/j.yjmcc.2015. 10.032

Maejima, Y., Kyoi, S., Zhai, P., Liu, T., Li, H., Ivessa, A., et al. (2013). Mst1 inhibits autophagy by promoting the interaction between Beclin1 and Bcl-2. Nat. Med. 19, 1478-1488. doi: 10.1038/nm.3322

Manning, W. J., Wei, J. Y., Katz, S. E., Litwin, S. E., and Douglas, P. S. (1994). In vivo assessment of LV mass in mice using high-frequency cardiac ultrasound: necropsy validation. Am. J. Physiol. 266, H1672-H1675. doi: 10.1152/ajpheart. 1994.266.4.H1672

Mao, S., Li, W., Qa'aty, N., Vincent, M., Zhang, M., and Hinek, A. (2016a). Tanshinone IIA inhibits angiotensin II induced extracellular matrix remodeling in human cardiac fibroblasts-Implications for treatment of pathologic cardiac remodeling. Int. J. Cardiol. 202, 110-117. doi: 10.1016/j.ijcard.2015.08.191

Mao, S., Li, X., Wang, L., Yang, P. C., and Zhang, M. (2015). Rationale and design of sodium tanshinone IIA sulfonate in left ventricular remodeling secondary to acute myocardial infarction (STAMP-REMODELING) trial: a randomized controlled study. Cardiovasc. Drugs Ther. 29, 535-542. doi: 10.1007/s10557015-6625-2
Mao, S., Wang, L., Ouyang, W., Zhou, Y., Qi, J., Guo, L., et al. (2016b). Traditional Chinese medicine, Danlou tablets alleviate adverse left ventricular remodeling after myocardial infarction: results of a double-blind, randomized, placebocontrolled, pilot study. BMC Complement. Altern. Med. 16:447. doi: 10.1186/ s12906-016-1406-4

Mao, S., Wang, Y., Zhang, M., and Hinek, A. (2014). Phytoestrogen, tanshinone IIA diminishes collagen deposition and stimulates new elastogenesis in cultures of human cardiac fibroblasts. Exp. Cell Res. 323, 189-197. doi: 10.1016/j.yexcr. 2014.02.001

Matsui, Y., Takagi, H., Qu, X., Abdellatif, M., Sakoda, H., Asano, T., et al. (2007). Distinct roles of autophagy in the heart during ischemia and reperfusion: roles of AMP-activated protein kinase and Beclin 1 in mediating autophagy. Circ. Res. 100, 914-922. doi: 10.1161/01.RES.0000261924.76669.36

Nadtochiy, S. M., Redman, E., Rahman, I., and Brookes, P. S. (2011a). Lysine deacetylation in ischaemic preconditioning: the role of SIRT1. Cardiovasc. Res. 89, 643-649. doi: 10.1093/cvr/cvq287

Nadtochiy, S. M., Yao, H., McBurney, M. W., Gu, W., Guarente, L., Rahman, I., et al. (2011b). SIRT1-mediated acute cardioprotection. Am. J. Physiol. Heart Circ. Physiol. 301, H1506-H1512. doi: 10.1152/ajpheart.00587.2011

Nakai, A., Yamaguchi, O., Takeda, T., Higuchi, Y., Hikoso, S., Taniike, M., et al. (2007). The role of autophagy in cardiomyocytes in the basal state and in response to hemodynamic stress. Nat. Med. 13, 619-624. doi: 10.1038/nm1574

Oche, B., Chen, L., Ma, Y. K., Yang, Y., Li, C. X., Geng, X., et al. (2016). Cryptotanshinone and wogonin up-regulate eNOS in vascular endothelial cells via ERalpha and down-regulate iNOS in LPS stimulated vascular smooth muscle cells via ERbeta. Arch. Pharm. Res. 39, 249-258. doi: 10.1007/s12272015-0671-y

Omland, T., Aakvaag, A., Bonarjee, V. V., Caidahl, K., Lie, R. T., Nilsen, D. W., et al. (1996). Plasma brain natriuretic peptide as an indicator of left ventricular systolic function and long-term survival after acute myocardial infarction. Comparison with plasma atrial natriuretic peptide and N-terminal proatrial natriuretic peptide. Circulation 93, 1963-1969. doi: 10.1161/01.CIR.93.11.1963

Ozcan, U., Ozcan, L., Yilmaz, E., Duvel, K., Sahin, M., Manning, B. D., et al. (2008). Loss of the tuberous sclerosis complex tumor suppressors triggers the unfolded protein response to regulate insulin signaling and apoptosis. Mol. Cell 29, 541-551. doi: 10.1016/j.molcel.2007.12.023

Pan, H., Li, D., Fang, F., Chen, D., Qi, L., Zhang, R., et al. (2011). Salvianolic acid A demonstrates cardioprotective effects in rat hearts and cardiomyocytes after ischemia/reperfusion injury. J. Cardiovasc. Pharmacol. 58, 535-542. doi: 10.1097/FJC.0b013e31822de355

Qi, J., Yu, J., Wang, L., Guo, L., Ma, S., Huang, D., et al. (2013). Tongguan capsule protects against myocardial ischemia and reperfusion injury in mice. Evid. Based Complement. Alternat. Med. 2013:159237. doi: 10.1155/2013/159237

Riquelme, J. A., Chavez, M. N., Mondaca-Ruff, D., Bustamante, M., Vicencio, J. M., Quest, A. F., et al. (2016). Therapeutic targeting of autophagy in myocardial infarction and heart failure. Expert Rev. Cardiovasc. Ther. 14, 1007-1019. doi: $10.1080 / 14779072.2016 .1202760$

Salminen, A., and Kaarniranta, K. (2009). SIRT1: regulation of longevity via autophagy. Cell. Signal. 21, 1356-1360. doi: 10.1016/j.cellsig.2009.02.014

Sharp, T. E., Schena, G. J., Hobby, A. R., Starosta, T., Berretta, R. M., Wallner, M., et al. (2017). Cortical bone stem cell therapy preserves cardiac structure and function after myocardial infarction. Circ. Res. 121, 1263-1278. doi: 10.1161/ CIRCRESAHA.117.311174

Shioi, T., McMullen, J. R., Tarnavski, O., Converso, K., Sherwood, M. C., Manning, W. J., et al. (2003). Rapamycin attenuates load-induced cardiac hypertrophy in mice. Circulation 107, 1664-1670. doi: 10.1161/01.CIR.0000057979.36322.88

Shirakabe, A., Ikeda, Y., Sciarretta, S., Zablocki, D. K., and Sadoshima, J. (2016). Aging and autophagy in the heart. Circ. Res. 118, 1563-1576. doi: 10.1161/ CIRCRESAHA.116.307474

Sin, T. K., Tam, B. T., Yung, B. Y., Yip, S. P., Chan, L. W., Wong, C. S., et al. (2015). Resveratrol protects against doxorubicin-induced cardiotoxicity in aged hearts through the SIRT1-USP7 axis. J. Physiol. 593, 1887-1899. doi: 10.1113/jphysiol. 2014.270101

Thapalia, B. A., Zhou, Z., and Lin, X. (2014). Autophagy, a process within reperfusion injury: an update. Int. J. Clin. Exp. Pathol. 7, 8322-8341.

Thomas, R. L., Roberts, D. J., Kubli, D. A., Lee, Y., Quinsay, M. N., Owens, J. B., et al. (2013). Loss of MCL-1 leads to impaired autophagy and rapid development of heart failure. Genes Dev. 27, 1365-1377. doi: 10.1101/gad.215871.113 
Velagaleti, R. S., Pencina, M. J., Murabito, J. M., Wang, T. J., Parikh, N. I., D'Agostino, R. B., et al. (2008). Long-term trends in the incidence of heart failure after myocardial infarction. Circulation 118, 2057-2062. doi: 10.1161/ CIRCULATIONAHA.108.784215

Wu, X., He, L., Chen, F., He, X., Cai, Y., Zhang, G., et al. (2014). Impaired autophagy contributes to adverse cardiac remodeling in acute myocardial infarction. PLoS One 9:e112891. doi: 10.1371/journal.pone.0112891

Yamamoto, T., and Sadoshima, J. (2011). Protection of the heart against ischemia/reperfusion by silent information regulator 1. Trends Cardiovasc. Med. 21, 27-32. doi: 10.1016/j.tcm.2012.01.005

Yan, L., Vatner, D. E., Kim, S. J., Ge, H., Masurekar, M., Massover, W. H., et al. (2005). Autophagy in chronically ischemic myocardium. Proc. Natl. Acad. Sci. U.S.A. 102, 13807-13812. doi: 10.1073/pnas.0506843102

Yang, J., Liu, X., Bhalla, K., Kim, C. N., Ibrado, A. M., Cai, J., et al. (1997). Prevention of apoptosis by $\mathrm{Bcl}-2$ : release of cytochrome $\mathrm{c}$ from mitochondria blocked. Science 275, 1129-1132. doi: 10.1126/science.275.5303.1129

Yuan, X., Jing, S., Wu, L., Chen, L., and Fang, J. (2014). Pharmacological postconditioning with tanshinone IIA attenuates myocardial ischemiareperfusion injury in rats by activating the phosphatidylinositol 3-kinase pathway. Exp. Ther. Med. 8, 973-977. doi: 10.3892/etm.2014.1820
Zhang, S., Tang, X., Tian, J., Li, C., Zhang, G., Jiang, W., et al. (2011) Cardioprotective effect of sulphonated formononetin on acute myocardial infarction in rats. Basic Clin. Pharmacol. Toxicol. 108, 390-395. doi: 10.1111/ j.1742-7843.2011.00676.x

Zhang, Y., Mi, S. L., Hu, N., Doser, T. A., Sun, A., Ge, J., et al. (2014). Mitochondrial aldehyde dehydrogenase 2 accentuates aging-induced cardiac remodeling and contractile dysfunction: role of AMPK, Sirt1, and mitochondrial function. Free Radic. Biol. Med. 71, 208-220. doi: 10.1016/j.freeradbiomed.2014.03.018

Conflict of Interest Statement: The authors declare that the research was conducted in the absence of any commercial or financial relationships that could be construed as a potential conflict of interest.

Copyright (c) 2018 Mao, Chen, Li, Guo and Zhang. This is an open-access article distributed under the terms of the Creative Commons Attribution License (CC BY). The use, distribution or reproduction in other forums is permitted, provided the original author(s) and the copyright owner are credited and that the original publication in this journal is cited, in accordance with accepted academic practice. No use, distribution or reproduction is permitted which does not comply with these terms. 